\title{
A method to determine fracture toughness using cube-corner indentation
}

\author{
Taihua Zhang, ${ }^{*}$ Yihui Feng, Rong Yang and Peng Jiang \\ State Key Laboratory of Nonlinear Mechanics (LNM), Institute of Mechanics, Chinese Academy of Sciences, Beijing 100190, China
}

Received 20 August 2009; revised 14 October 2009; accepted 20 October 2009

Available online 23 October 2009

For the cube-corner indenter, an approximate linear relationship between the ratio of hardness $(H)$ to reduced modulus $\left(E_{r}\right)$ and the ratio of unloading work $\left(W_{u}\right)$ to total loading work $\left(W_{t}\right)$ is confirmed by finite-element calculations and by experiments. Based on this relationship a convenient method to determine the fracture toughness $\left(K_{I C}\right)$ of brittle materials, especially for those at small scale, using cube-corner indentations is proposed. Finally, the method is calibrated by indentation experiments on a set of brittle materials.

(C) 2009 Acta Materialia Inc. Published by Elsevier Ltd. All rights reserved.

Keywords: Fracture; Toughness; Cube-corner indentation

With the development of nanostructured materials and the miniaturization of electromechanical devices, the measurement of mechanical properties, including fracture toughness, of small volumes or thin films of materials is in demand. For the determination of fracture toughness at a small scale, which is of interest in this paper, and cannot be achieved by conventional methods [1-3], the indentation test using a sharp pyramidal indenter may be a feasible solution. A well-known formula exists for determining the fracture toughness of brittle materials using indentation tests:

$K_{I C}=\delta\left(\frac{E}{H}\right)^{1 / 2} \frac{F_{m}}{c^{3 / 2}}$,

which was initially developed by Lawn et al. [4] for indentation tests using the Vickers indenter, a four-sided pyramid, on the assumption that half-penny cracks predominate. In Eq. (1), $E$ and $H$ are the elastic modulus and the hardness of the test material, respectively, $F_{m}$ is the peak load of the indentation cycle, $c$ is the length of the radial crack trace on the material surface after the indenter withdrawing, and $\delta$ is an empirical constant independent of the material. Other researchers [5-7] have shown that Eq. (1) is effective when radial cracks other than half-penny cracks predominate or other pyramidal indenters such as Berkovich (three-sided,

\footnotetext{
*Corresponding author. Fax: +8610 82543977; e-mail: zhangth@ lnm.imech.ac.cn
}

having the same depth-projected area ratio as a Vickers indenter) and cube-corner (three-sided, in the shape of the corner of a cube) are used. The parameter, $\delta$, takes different values for different indenters: 0.016 for Vickers and Berkovich [6,8]; 0.032-0.040 for cube-corner [6,7,9]. Compared with Vickers and Berkovich, the cube-corner indenter can reduce cracking thresholds by 1-2 orders due to its much sharper included angle [7]. Therefore the cube-corner indenter is more suitable for determining the fracture toughness of brittle materials at a small scale. However, the latest cube-corner indentation method [7] developed has two shortcomings. Firstly, the test procedure is complex as two kinds of indenters are needed: a cube-corner indenter to produce radial cracks, and a Berkovich indenter to measure $E$ and $H$ according to standard nanoindentation techniques [10]. Secondly, there may be significant errors in measuring $E$ and $H$ when "pile-up" occurs.

In this paper, the approximate linear relationship between the ratio of hardness $H$ to reduced modulus $E_{r}$ and the ratio of unloading work $W_{u}\left(W_{u}=\int_{h_{m}}^{h_{m}} F d h-\right.$ see Figure 1a and Refs. [11-13]) to total loading work $W_{t}\left(W_{t}=\int_{0}^{h_{m}} F d h\right.$ - see Figure 1a and Refs. [11-13]) for cube-corner indentations is confirmed by finite-element method (FEM) calculations and by experiment. Based on this relationship, a method for determining fracture toughness at a small scale using a single cubecorner indenter is proposed. While taking advantage of the low cracking thresholds of the cube-corner indenter, this method can avoid the two shortcomings 

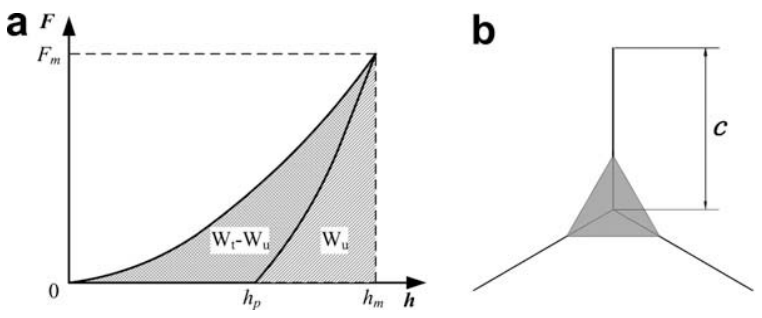

Figure 1. Analyzing parameters of the proposed method: (a) peak load $\left(F_{m}\right)$, unloading work $\left(W_{u}\right)$, total loading work $\left(W_{t}\right)$; (b) length of radial crack $(c)$.

mentioned above. Firstly, only the cube-corner indenter is used, which simplifies the test procedure. Secondly, $W_{u}$ and $W_{t}$, instead of $E$ and $H$, are the input parameters, and the use of these can avoid the possible errors in determining $E$ and $H$ when pile-up occurs.

There has been much work $[11,12,14]$ on estimating the relationship between $H / E_{r}$ and $W_{u} / W_{t}$ for geometrically self-similar indenters. The approximate linear relationship between $H / E_{r}$ and $W_{u} / W_{t}$ for indenters with certain included angles or for several kinds of materials has been confirmed. However, it is still unclear weather there is also such an approximate linear relationship for the combination of the cube-corner indenter and brittle materials, especially when friction between the indenter and the material surface is considered.

For this reason, extensive FEM calculations were carried out using ABAQUS [15] to examine the relationship between $H / E_{r}$ and $W_{u} / W_{t}$ for the cube-corner indenter. In the FEM model, the cube-corner indenter is simplified as a rigid conical indenter with its half-included angle, $\alpha=42.3^{\circ}$. The stress-strain $(\sigma-\varepsilon)$ relationship of the examined materials under uniaxial tension is assumed to obey:

$$
\begin{array}{ll}
\sigma=E \varepsilon & \text { for } \varepsilon \leqslant Y / E, \\
\sigma=Y(E / Y)^{n} \varepsilon^{n} & \text { for } \varepsilon \geqslant Y / E,
\end{array}
$$

where $Y$ is initial yield stress and $n$ is the work-hardening exponent. In the calculations, $n$ is assumed to be 0.0 , $0.1,0.3$ and 0.5 . For each $n, Y / E$ varies from 0.001 to 0.1 . For all combinations between $n$ and $Y / E$, Poisson's ratio, $v$, is assumed to be 0.25 . The friction coefficient, $f$, between the indenter and examined materials is assumed to be 0.15 . The reduced modulus $E_{r}$ is calculated by:

$\frac{1}{E_{r}}=\frac{1-v_{i}^{2}}{E_{i}}+\frac{1-v^{2}}{E}$,

where $E_{i}$ and $v_{i}$ are the elastic modulus and Poisson's ratio of the indenter, respectively. If the indenter is considered rigid in the calculations, Eq. (3) reduces to:

$\frac{1}{E_{r}}=\frac{1-v^{2}}{E}$.

Hardness, $H$, is calculated by:

$H=\frac{F_{m}}{A_{c}}$,

where $F_{m}$ is the peak indenter load, and $A_{c}$ is the projected contact area. $W_{u}$ and $W_{t}$ are calculated by integrating the unloading and loading part of the indentation load-depth $(F-h)$ curve, respectively. $H / E_{r}$ and $W_{u} / W_{t}$ for every combination of $n, Y / E, v$ and $f$ are plotted in Figure 2.

To assess the validation of the FEM calculations, cube-corner and Berkovich indentation tests using a Nano Indenter XP (Agilent Technologies, Oak Ridge, $\mathrm{TN}$ ) were carried out on three brittle materials: fused silica, Pyrex 7740 glass and $\mathrm{ZnS}$. Berkovich indentation tests were performed to measure $E$ of the test materials as $E$ is essentially independent of the indenter used. The details are according to ISO14577 [13]. $E_{r}$ is calculated using Eq. (3) with $E_{i}=1140 \mathrm{GPa}$ and $v_{i}=0.07$. $H / E_{r}$ and $W_{u} / W_{t}$ of each material are also plotted in Figure 2. It can be seen that there is an approximate linear relationship:

$\frac{H}{E_{r}}=k \frac{W_{u}}{W_{t}}$,

for the cube-corner indenter. Least-squares fitting on the FEM calculation results data yields $k \approx 0.527$.

As the elastic moduli of most brittle materials are much smaller than that of the diamond indenter, Eq. (4) also works well for most brittle material-diamond indenter combinations. Furthermore, the Poisson's ratios of most brittle materials are $\sim 0.25$. Inserting Eqs. (4) and (6) into Eq. (1) with assuming $v=0.25$, $k=0.527$, we can obtain the formula for the method proposed in this paper:

$K_{I C}=\lambda\left(\frac{W_{u}}{W_{t}}\right)^{-1 / 2} \frac{F_{m}}{c^{3 / 2}}$,

where $W_{u}$ and $W_{t}$ can be calculated by integrating the unloading and loading data of the $F-h$ curve of the cube-corner indentation test, respectively, $F_{m}$ can be obtained on the $F-h$ curve, $c$ can be measured by microscopy (see Fig. 1b), and $\lambda$ is a constant number independent of the material. It should be noted that the hardness $H$ in Eq. (1) must be determined using the cube-corner indenter rather than the Vickers indenter [6] or the Berkovich indenter [7], i.e. $\delta$ in Eq. (1) should be a different constant number between 0.032 and 0.040 when inserting Eqs. (4) and (6) into Eq. (1). Hence, the constant $\lambda$ must be determined by calibrating Eq. (7) by indentation tests on a set of brittle materials for which $K_{I C}$ is known.

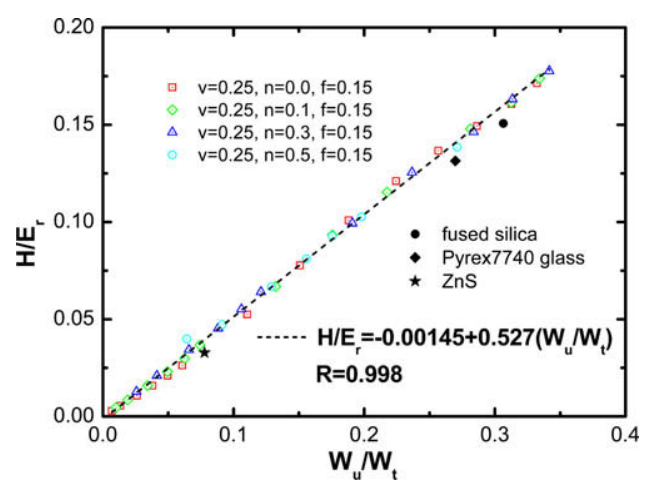

Figure 2. Relationship between $H / E_{r}$ and $W_{u} / W_{t}$ for the cube-corner indenter. 
Table 1. $K_{I C}$ of test materials for calibration of Eq. (7).

\begin{tabular}{ll}
\hline Material & $K_{I C}(\mathrm{MPa} \cdot \sqrt{\mathrm{m}})$ \\
\hline Fused silica & $0.58^{\mathrm{a}}$ \\
Soda-lime glass & $0.75^{\mathrm{b}}$ \\
Pyrex7740 glass & $0.63^{\mathrm{b}}$ \\
Si $\left(\begin{array}{lll}1 & 1 & 1\end{array}\right)$ & $0.7^{\mathrm{a}}$ \\
\hline
\end{tabular}

${ }^{a}$ Ref. [7].

${ }^{\mathrm{b}}$ Ref. [8].

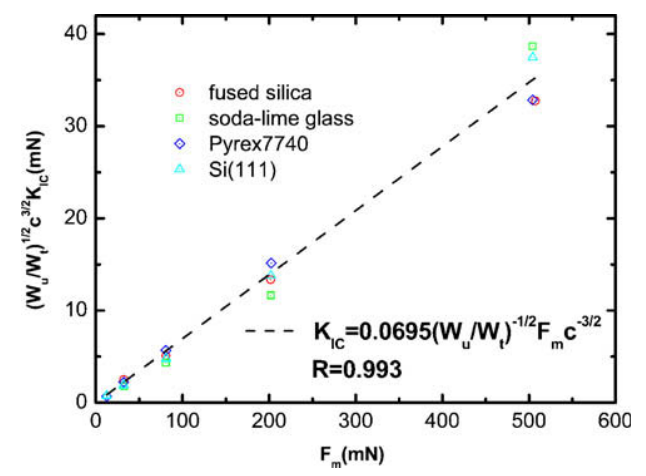

Figure 3. Calibration of the proposed method by experiments on brittle materials.

To calibrate Eq. (7), four brittle materials - fused silica, soda-lime glass, Pyrex7740 glass and $\mathrm{Si}\left(\begin{array}{lll}1 & 1 & 1\end{array}\right)$-were chosen as test materials. The indentation tests were carried out using a Nano Indenter XP. The fracture toughness of the test materials are obtained from Refs. [7,8] (see Table 1). For each test material, five peak loads, $F_{m}=12.5,32,80,200$ and $500 \mathrm{mN}$, were applied. The radial crack lengths were measured using scanning electron microscopy (SEM). The data of $\left(W_{u} / W_{t}\right)^{1 / 2} c^{3 / 2} K_{I C}$ and $F_{m}$ are plotted in Figure 3. When measuring radial crack length, $c$, the radial cracks affected by chipping in the material surface are excluded. For each material, $W_{u} / W_{t}$ with $F_{m}=12.5 \mathrm{mN}$ is used for all five peak loads because indentation curves $(F-h)$ are smooth for all materials when $F_{m}=12.5 \mathrm{mN}$. By least-squares fitting of the test data in Figure 3, we can obtain $\lambda \approx 0.0695$ for Eq. (7).

In addition to the calibration of Eq. (7), we also found some interesting results from the indentation test observations. Firstly, the emergence of discontinuity on an $F-h$ curve always accompanies obvious chipping in the material surface according to the post-indentation SEM observation, whereas no such chipping is observed for a smooth $F-h$ curve. Secondly, for a certain material, $W_{u} / W_{t}$ with different peak loads do not vary much if their $F-h$ curves demonstrate no obvious discontinuity.
Therefore we suggest that the indentation tests with smooth $F-h$ curves make sense when using Eq. (7).

In summary, we established, by FEM calculations and by experiments, an approximate linear relationship of $H / E_{r}$ and $W_{u} / W_{t}$ for the cube-corner indenter when friction is considered. Based on the linear relationship, we proposed a method to determine fracture toughness of brittle materials using a single cube-corner indenter, and calibrated the method by experiments on a set of brittle materials. The method proposed can simplify the test procedure while greatly reducing the cracking thresholds. The method uses the readily measurable $W_{u}$ and $W_{t}$, instead of $E$ and $H$, as input parameters. In the future, more brittle materials should be studied to assess the effectiveness of Eq. (7). The effects of crack systems induced by different peak loads should also be studied in detail. These studies are in process.

The authors thank Professor Yang-Tse Cheng, Professor Che-Min Cheng, Dr. Yong Huan, Xin Jiang for helpful discussions. The help of Dr. Jijia Xie and Guihua Duan with SEM observations is also appreciated. We acknowledge the support of the NSFC (10872200, 10721202, 10572142).

[1] ISO 12737:2005, Metallic Materials-Determination of Plane-Strain Fracture Toughness.

[2] ASTM E 399-06, Standard Test Method for LinearElastic Plane-Strain Fracture Toughness $\mathrm{K}_{\mathrm{IC}}$ of Metallic Materials.

[3] ASTM C 1421-01b, Standard Test Methods for Determination of Fracture Toughness of Advanced Ceramics at Ambient Temperature.

[4] B.R. Lawn, A.G. Evans, D.B. Marshall, J. Am. Ceram. Soc. 63 (1980) 574.

[5] M.T. Laugier, J. Mater. Sci. Lett. 6 (1985) 355.

[6] G.M. Pharr, D.S. Harding, W.C. Oliver, in: M. Nastasi, D.M. Parkin, H. Gleier (Eds.), Mechanical Properties and Deformation Behavior of Materials Having UltraFine Microstructures, Kluwer, Dordrecht, 1993, pp. 449461.

[7] D.S. Harding, W.C. Oliver, G.M. Pharr, Mater. Res. Soc. Symp. Proc. 356 (1995) 663.

[8] G.R. Anstis, P. Chantikul, B.R. Lawn, D.B. Marshall, J. Am. Ceram. Soc. 61 (1981) 533.

[9] G.M. Pharr, Mater. Sci. Eng. A253 (1998) 151.

[10] W.C. Oliver, G.M. Pharr, J. Mater. Res. 7 (1992) 1564.

[11] Y.-T. Cheng, C.-M. Cheng, Appl. Phys. Lett. 73 (1998) 614.

[12] R. Yang, T.H. Zhang, P. Jiang, Y.L. Bai, Appl. Phys. Lett. 92 (2008) 231906.

[13] ISO 14577:2002, Metallic Materials: Instrumented Indentation Test for Hardness and Materials Parameters.

[14] Y.-T. Cheng, Z. Li, C.-M. Cheng, Phil. Mag. A82 (2002) 1821.

[15] HKS, Inc., Pawtucket, RI, USA. 